\title{
Primary Visual Cortex
}

National Cancer Institute

\section{Source}

National Cancer Institute. Primary Visual Cortex. NCI Thesaurus. Code C97340.

A brain region in the occipital cortex that receives visual stimuli from the retina. 